\title{
Tissue factor pathway inhibitor-2 is a novel inhibitor of matrix metalloproteinases with implications for atherosclerosis
}

\author{
Michael P. Herman, ${ }^{1}$ Galina K. Sukhova, ${ }^{1}$ Walter Kisiel, ${ }^{2}$ Don Foster, ${ }^{3}$ Marilyn R. Kehry, \\ Peter Libby, ${ }^{1}$ and Uwe Schönbeck ${ }^{1}$ Brigham and Women's Hospital, Harvard Medical School, Boston, Massachusetts, USA
${ }^{2}$ Department of Pathology, School of Medicine, University of New Mexico, Albuquerque, New Mexico, USA
${ }^{3}$ Zymogenetics Inc., Seattle, Washington, USA \\ ${ }^{1}$ Leducq Center for Cardiovascular Research, Division of Cardiovascular Medicine, Department of Medicine, \\ ${ }^{4}$ Boehringer Ingelheim Pharmaceuticals Inc., Ridgefield, Connecticut, USA
}

Address correspondence to: Uwe Schönbeck, Leducq Center for Cardiovascular Research, Division of Cardiovascular Medicine, Department of Medicine, Brigham and Women's Hospital, Harvard Medical School, 221 Longwood Avenue, BLI 448, Boston, Massachusetts 02115, USA. Phone: (617) 278-0455; Fax: (617) 732-6961; E-mail: uschoenbeck@rics.bwh.harvard.edu.

Marilyn R. Kehry's present address is: IDEC Pharmaceuticals Corp., San Diego, California, USA.

Received for publication May 23, 2000, and accepted in revised form March 23, 2001.

\begin{abstract}
Degradation of ECM, particularly interstitial collagen, promotes plaque instability, rendering atheroma prone to rupture. Previous studies implicated matrix metalloproteinases (MMPs) in these processes, suggesting that dysregulated MMP activity, probably due to imbalance with endogenous inhibitors, promotes complications of atherosclerosis. We report here that the serine proteinase inhibitor tissue factor pathway inhibitor-2 (TFPI-2) can function as an MMP inhibitor. TFPI-2 diminished the ability of the interstitial collagenases MMP-1 and MMP-13 to degrade triple-helical collagen, the primary load-bearing molecule of the ECM within human atheroma. In addition, TFPI-2 also reduced the activity of the gelatinases MMP-2 and MMP-9. In contrast to the "classical" tissue inhibitors of MMPs (TIMPs), TFPI-2 expression in situ correlated inversely with MMP levels in human atheroma. TFPI-2 colocalized primarily with smooth muscle cells in the normal media as well as the plaque's fibrous cap. Conversely, the macrophage-enriched shoulder region, the prototypical site of matrix degradation and plaque rupture, stained only weakly for TFPI-2 but intensely for gelatinases and interstitial collagenases. Evidently, human mononuclear phagocytes, an abundant source of MMPs within human atheroma, lost their ability to express this inhibitor during differentiation in vitro. These findings establish a new, anti-inflammatory function of TFPI-2 of potential pathophysiological significance for human diseases, including atherosclerosis.
\end{abstract}

J. Clin. Invest. 107:1117-1126 (2001).

\section{Introduction}

Despite its name, tissue factor pathway inhibitor-2 (TFPI-2) poorly inhibits tissue factor (TF) and as yet has no defined physiologic function. Originally cloned from a human placental cDNA library while seeking Kunitz-inhibitory domain proteins related to TFPI-1, TFPI-2's sequence matched that of placental protein 5 (PP5) and matrix serine protease inhibitor (MSPI), described previously in dermal fibroblasts as well as in placental and endothelial cells (ECs) (1-3). Although a molecular weight of $25 \mathrm{kDa}$ was originally proposed for the unglycosylated protein, immunoreactive TFPI-2 typically migrates with an apparent molecular weight of 27,31 , and $33 \mathrm{kDa}$ due to differential glycosylation $(1,4)$. Cloning of TFPI-2 revealed similarities in the overall domain organization as well as considerable amino acid sequence homology with TFPI-1, an important regulator of the extrinsic pathway of blood coagulation via its inhibition of factor $\mathrm{Xa}$ and factor
VIIa/TF complex (1). Nevertheless, TFPI-2 only weakly inhibits these coagulation proteins. Though established targets of TFPI-2 include certain other serine proteases, e.g., kallikrein, trypsin, chymotrypsin, and plasmin, its biological function remains uncertain (1, 2, 5). Curiously, most of the TFPI-2 expressed by ECs of various origins localizes within the ECM (4).

Turnover of the arterial ECM contributes crucially to a variety of diseases, including atherosclerosis (6). Interstitial collagen, comprising up to $60 \%$ of the total protein of plaques, confers stability on the lesion's fibrous cap that separates the procoagulant lipid core from the blood $(7,8)$. In plaques that have ruptured, the fibrous cap of the plaque's shoulder region, the prototypical site of rupture, has particularly sparse collagen $(9,10)$. Thus, the balance between matrix conservation and matrix degradation probably determines plaque stability and thereby the risk of acute clinical complications such as myocardial infarction and stroke. 
A variety of proteases, including the matrix metalloproteinases (MMPs), degrade matrix macromolecules. The MMP family encompasses at least 27 members that share common structural elements and are typically released as inactive zymogens (11). Inflammatory cytokines, such as interleukin-1 (IL-1), TNF- $\alpha$, and CD40 ligand (CD40L), induce the expression of MMPs in various cell types, including ECs, smooth muscle cells (SMCs), and mononuclear phagocytes (MØs) (10-16). Attaining matrix-degrading activity requires maturation of the MMP zymogen $(17,18)$.

However, the expression of mature MMPs does not necessarily correlate with matrix-degrading activity since endogenous inhibitors tightly regulate MMP activity. Such endogenous inhibitors were considered restricted to the family of tissue inhibitors of MMPs (TIMPs) (19-25). Interestingly, expression of this prototypical class of inhibitors does not correlate inversely with enhanced MMP activity in situ, as would be expected with increased matrix turnover $(26,27)$. Additionally, certain TIMPs (e.g., TIMP-2) are implicated in the membrane-type metalloproteinase-mediated (MTMMP-mediated) activation of distinct MMP family members (e.g., MMP-2 and MMP-13), as well as in the release of active MMPs $(28,29)$. Thus, operation of inhibitory mechanisms beyond TIMPs has been postulated in atheroma, although simple quantitative correlation of MMP to TIMP probably does not adequately reflect complex in vivo situations (e.g., local concentrations of matrix-degrading enzymes and their inhibitors might vary due to compartmentalization; see refs 30-32). Interestingly, previous work from several groups implicated members of the serpin superfamily, i.e., $\alpha_{2}$-macroglobulin and RECK, in the regulation of MMP activity (33-35).

In accordance with its prominent ECM localization (4), previous reports suggested a matrix-protective function for the serpin TFPI-2. TFPI-2 inhibited matrix degradation and invasion by fibrosarcoma cells (36). Rao et al. demonstrated that TFPI- 2 concentration dependently inhibited plasmin-dependent activation of pro-MMP-1 and pro-MMP-3, though it was unclear whether inhibition occurred because of binding to plasmin or to MMPs (5). We hypothesized here that TFPI-2 might act directly as an endogenous inhibitor of active MMPs and that local TFPI-2 deficiency may pertain to human atheroma.

\section{Methods}

Materials. Rabbit anti-human TFPI-2 Ab was prepared as described previously (1). Recombinant human TFPI2 was expressed in hamster kidney cells transfected with TFPI-2 cDNA and purified from serum-free conditioned medium by a combination of heparin-agarose chromatography, Mono Q FPLC, Mono S FPLC, and Superose 12 FPLC (all from Amersham Pharmacia Biotech Inc., Piscataway, New Jersey, USA). Purified TFPI-2 migrated as a single band with an apparent molecular weight of $32 \mathrm{kDa}$ in SDS-PAGE under reduc- ing (heat-inactivated) and nonreducing (non-heattreated) conditions (Figure 1h). The amino-terminal sequence of recombinant TFPI-2 was identical to that predicted from the cDNA. Human recombinant IL-1 $\beta$ and TNF- $\alpha$ were obtained from Endogen Inc.(Cambridge, Massachusetts, USA), and recombinant TIMP-1 was purchased from Calbiochem-Novabiochem Corp. (La Jolla, California, USA). Human recombinant CD40L was generated as described previously (37). Escherichia coli endotoxin (LPS) was purchased from Sigma Chemical Co. (St. Louis, Missouri, USA). MMP-2 zymogen was purchased from Oncogene Research Products (San Diego, California, USA). Polyclonal rabbit anti-human MMP-1, -2, and -9 (Triple Point Biologics Inc., Portland, Oregon, USA) as well as mouse anti-human MMP-13 (kindly provided by Carlos Lopez-Otin, University of Oviedo, Oviedo, Spain) were used for immunohistochemistry and Western blot analysis. Monoclonal mouse anti-human TIMP-1 and TIMP-2 were obtained from Calbiochem-Novabiochem Corp. Human recombinant cathepsin $\mathrm{S}$ and cathepsin $\mathrm{K}$ were kindly provided by Guo Ping-Shi (University of California, San Francisco, California, USA).

MMP-activity assays. Assays of MMP and cathepsin activity employed either purified triple-helical type I collagen (Chemicon International, Temecula, California, USA) or self-quenching fluorophore-conjugated native or synthetic substrates (Molecular Probes Inc., Eugene, Oregon, USA; Chondrex LLC, Redmond, Washington, USA; or Chemicon International). Analysis of MMP-1 and MMP-13 activity (both $10 \mu \mathrm{g} / \mathrm{ml}$, provided as truncated $41 / 44-\mathrm{kDa}$ and $27-\mathrm{kDa}$ forms, respectively; Chemicon International) required activation via $1 \mathrm{mM}$ p-aminophenylmercuric acetate (APMA) for 2 hours. MMP-2 and MMP-9 (both $10 \mu \mathrm{g} / \mathrm{ml}$; Oncogene Research Products) were provided as mature, active enzymes (66 kDa and $83 \mathrm{kDa}$, respectively). To analyze inhibition, MMPs were preincubated with the respective concentrations of TFPI- 2 for 1 hour at $37^{\circ} \mathrm{C}$ in reaction buffer $(50$ mM Tris-HCl, pH 7.6, 150 mM NaCl, 5 mM $\mathrm{CaCl}_{2}, 1 \mu \mathrm{M}$ zinc chloride, $0.01 \%$ vol/vol BRIJ 35). The time required for equilibration (30 minutes) was determined by following the progress of inhibition after mixing MMP and TFPI-2. Subsequently, the respective FITC-conjugated substrate was added $\left(25^{\circ} \mathrm{C}\right)$ and enzymatic activity determined during the linear rate of the reaction by continuous monitoring with a fluorescence spectrophotometer at excitation and emission wavelengths of $495 \mathrm{~nm}$ and $515 \mathrm{~nm}$, respectively. $\mathrm{EC}_{50}$ values were determined for the incubation of each of the respective MMPs with TFPI-2. Reactions employing nonconjugated collagen were analyzed by Western blotting with an anti-type I collagen Ab. Inhibitory activity of TFPI- 2 against cathepsins was investigated by applying cathepsin S or $\mathrm{K}$ (both $10 \mu \mathrm{g} / \mathrm{ml}$ ) to processing assays identical in procedure to those described above employing an acidic protease processing buffer $(0.05 \%$ Triton X-100, $20 \mathrm{mM}$ sodium acetate, and $1 \mathrm{mM}$ EDTA, $\mathrm{pH}$ 5.5), as described previously (38). 
$\mathbf{a}$

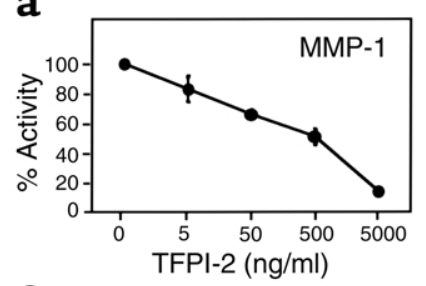

C

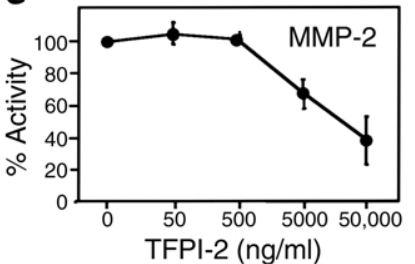

e
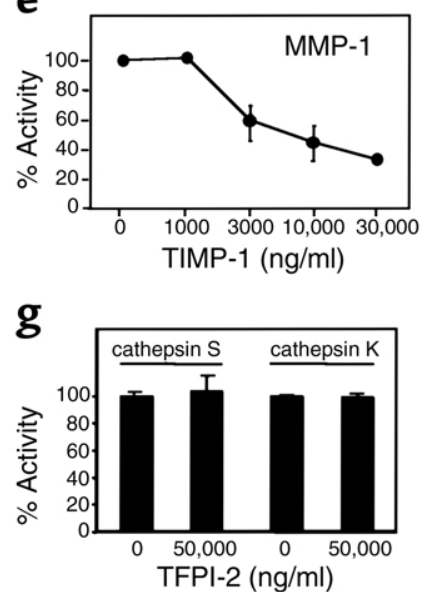

Figure 1

Human recombinant TFPI-2 (recTFPI-2) inhibits MMP activity. Human recombinant MMP-1 (a, e), MMP-13 (b, f), MMP-2 (c), MMP-9 (d), and cathepsin $\mathrm{S}$ or $\mathrm{K}(\mathbf{g})$ (all at $10 \mu \mathrm{g} / \mathrm{ml}$ ) were incubated with the respective concentrations of TFPI-2 (a-d, g) or TIMP-1 (e, f) (1 hour, $37^{\circ} \mathrm{C}$ ), and the ability of the enzymes to process fluorophore-conjugated native $(\mathbf{a}, \mathbf{b}, \mathbf{e}, \mathbf{f})$ or peptide $(\mathbf{c}, \mathbf{d}, \mathbf{g})$ substrate was measured as described. Percentage of activity was normalized to the activity obtained with the respective MMP lacking TFPI-2. Data shown represent mean \pm SD and are representative of at least three independent experiments. (h) Human recombinant TFPI-2 (10 $\mu \mathrm{g} /$ lane), expressed and purified as described in Methods, was applied to $15 \%$ SDS-PAGE analysis under reducing (Red.) and nonreducing (Non-Red.) conditions. Proteins were visualized by silver staining. The positions of the molecular-weight markers are indicated on the left.

The percentage of MMP/cathepsin activity was determined by dividing sample fluorescence intensity by the value obtained with identical concentrations of MMP without TFPI-2. For control purposes, TFPI-2 was preincubated $\left(1\right.$ hour, $\left.37^{\circ} \mathrm{C}\right)$ with trypsin (Sigma Chemical Co.) or VIIa/TF (American Diagnostica Inc., Greenwich, Connecticut, USA) or was replaced by either recombinant TFPI-1 (American Diagnostica Inc.) or TIMP-1. Additionally, heat-inactivated TFPI-2 (10 minutes, $95^{\circ} \mathrm{C}$ ) was employed. Data are presented as mean $\pm \mathrm{SD}$, obtained from duplicate determinations.

Western blot analysis. Samples were separated by standard SDS-PAGE under nonreducing conditions and blotted to polyvinylidene difluoride membranes (BioRad Laboratories Inc., Hercules, California, USA) using a semi-dry blotting apparatus $\left(0.8 \mathrm{~mA} / \mathrm{cm}^{2}, 30\right.$ minutes; Bio-Rad Laboratories Inc.). Blots were blocked (>2 hours) in 5\% defatted dry milk/PBS/0.1\% Tween-20. After 1 hour of incubation with the primary $\mathrm{Ab}$, blots were washed three times (PBS/0.1\% Tween), and the secondary, peroxidase-conjugated $\mathrm{Ab}$ (Jackson ImmunoResearch, West Grove, Pennsylvania, USA) was added for another hour. Finally, the blots were washed three times (PBS $/ 0.1 \%$ Tween) and immunoreactive proteins were visualized using Western blot chemiluminescent detection (NEN Life Science Products Inc., Boston, Massachusetts, USA).

Cell isolation and culture. Human vascular SMCs were isolated from human saphenous veins by explant outgrowth, and cultured in DMEM (BioWhittaker Inc., Walkersville, Maryland, USA) supplemented with $1 \%$ L-glutamine (BioWhittaker Inc.), $1 \%$ penicillin/streptomycin (BioWhittaker Inc.), and 10\% FBS (Atlanta Biologicals Inc., Norcross, Georgia, USA). SMCs were cultured 24 hours before the experiment in insulin/transferrin (IT) media lacking FBS, as described previously (39). SMCs were routinely characterized by immunostaining with anti-SMC $\alpha$-actin Ab (Enzo Diagnostics Inc., Farmingdale, New York, USA).

Mononuclear phagocytes were isolated by densitygradient centrifugation and subsequent counterflow elutriation from freshly prepared human PBMCs obtained from leukopacs of healthy donors. The purity of mononuclear phagocytes was equal to or greater than $96 \%$, as determined by FACS analysis (antihuman CD68 mAb FITC; PharMingen, San Diego, California, USA). Cells were cultured in RPMI-1640 medium (BioWhittaker Inc.) containing 5\% human serum (Sigma Chemical Co.). Twenty-four hours before stimulation, cells were incubated in serum-free RPMI-1640 medium.

Culture media and FBS contained less than $40 \mathrm{pg} / \mathrm{ml}$ endotoxin as determined by chromogenic Limulus amoebocyte assay (QLC-1000; BioWhittaker Inc.).

Biochemical analysis of human atherosclerotic lesions. Frozen tissue from five nonatherosclerotic aortae and carotid arteries and 12 carotid plaques were dichotomized into fibrous and atheromatous plaques by morphological criteria as described previously (9). The specimens were homogenized (Ultra-turrax T 25; IKA-Labortechnik, Wilmington, North Carolina, USA), lysed ( $0.3 \mathrm{~g}$ tissue $/ \mathrm{ml}$ in $10 \mathrm{mM}$ sodium phosphate, $150 \mathrm{mM} \mathrm{NaCl}, 1 \%$ Triton $\mathrm{X}-100,0.1 \%$ SDS, $0.5 \%$ sodium deoxycholate, $0.2 \%$ sodium azide), clarified $(13,000 \mathrm{~g}, 15$ minutes), and the protein concentration determined using a bicinchoninic acid (BCA) protein assay (Pierce Chemical Co., Rockford, Illinois, USA). Fifty micrograms total protein were applied to Western blot analysis.

Immunoprecipitation and zymography. Supernatants of SMC cultures, or the respective recombinant MMP preincubated with TFPI- $2\left(1\right.$ hour, $\left.37^{\circ} \mathrm{C}\right)$, were incu- 

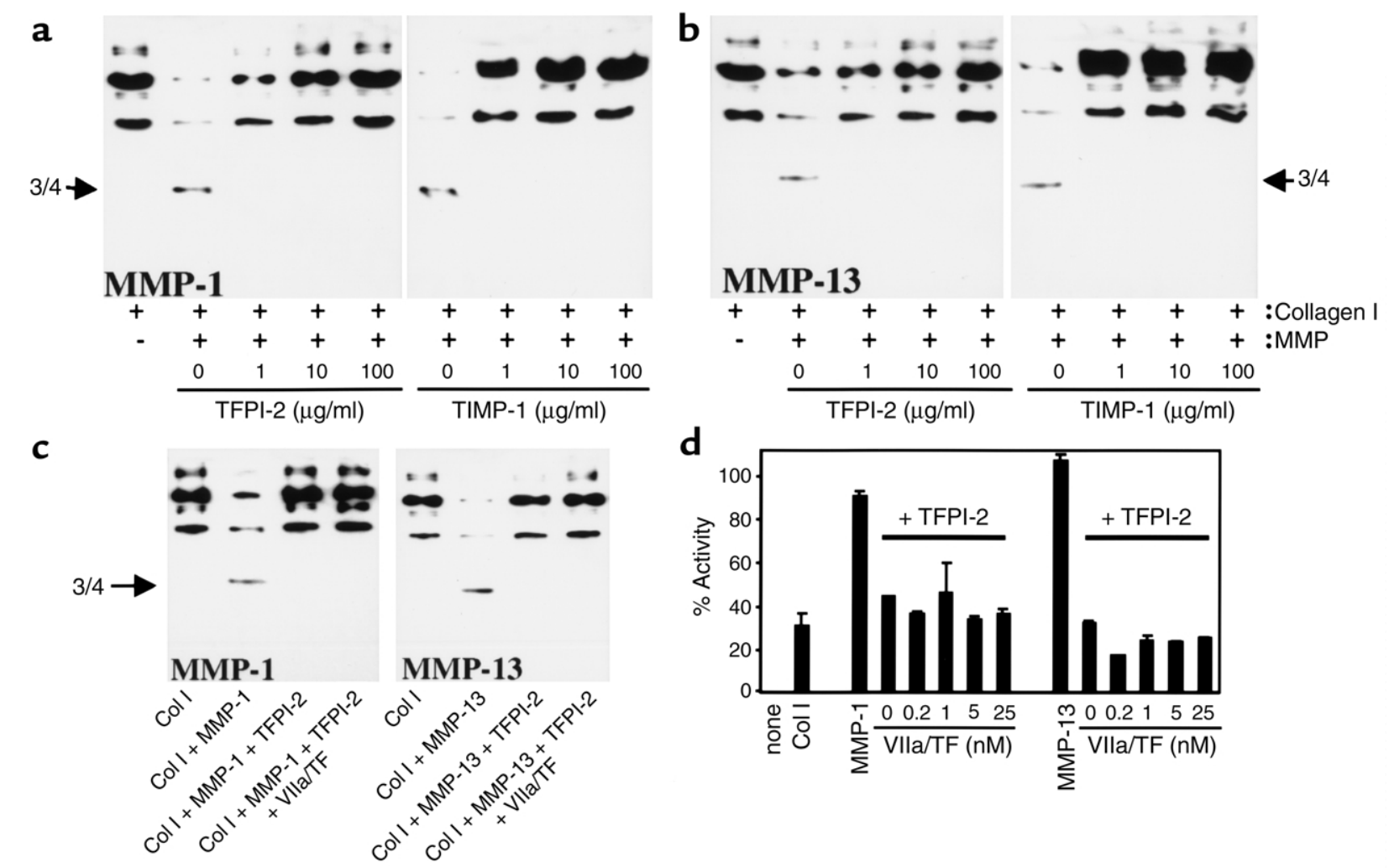

\section{Figure 2}

TFPI-2 inhibits processing of triple-helical type I collagen ( $\mathrm{Col}$ I) by interstitial collagenases. Purified human triple-helical type I collagen $(100 \mu \mathrm{g} / \mathrm{ml})$ was incubated $\left(24\right.$ hours, $\left.25^{\circ} \mathrm{C}\right)$ with either MMP- 1 (a) or MMP-13 (b) (both at $\left.10 \mu \mathrm{g} / \mathrm{ml}\right)$ preincubated with the respective concentration of TFPI-2 or TIMP-1, or (c) incubated with MMP-1 and MMP-13 (both at $10 \mu \mathrm{g} / \mathrm{ml}$ ) preincubated with TFPI-2 (100 $\mu \mathrm{g} / \mathrm{ml})$ complexed $\left(45\right.$ minutes, $\left.37^{\circ} \mathrm{C}\right)$ with VIla/TF $(20 \mathrm{nM})$. Reactions were applied to Western blot analysis with anti-type I collagen Ab. (d) Human recombinant MMP-1 or MMP-13 (both at $10 \mu \mathrm{g} / \mathrm{ml}$ ) were incubated with TFPI-2 $(100 \mu \mathrm{g} / \mathrm{ml}$ ) complexed with the respective concentrations of VIla/TF, and the subsequent ability of the MMPs to process native fluorogenic substrate was measured as described. Percentage of activity was normalized to the activity obtained with the respective MMP lacking TFPI-2. Data shown represent mean \pm SD and are representative of at least three independent experiments.

bated with either rabbit anti-human TFPI-2 $\mathrm{Ab}$ or nonimmunized rabbit IgG $\left(18\right.$ hour, $\left.4^{\circ} \mathrm{C}\right)$, and precipitated by subsequent addition of goat anti-rabbit IgG (18 hour, $4^{\circ} \mathrm{C}$; Jackson ImmunoResearch Laboratories Inc.) and protein A-Sepharose beads $\left(2\right.$ hours, $4^{\circ} \mathrm{C}$; Pharmacia Biotech Inc., Piscataway, New Jersey, USA). The precipitates and the original supernatants were separated by SDS-PAGE and applied to either zymography or Western blot analysis. For zymographic analysis, gels were washed twice (15 minutes) with $2.5 \%$ Triton $\mathrm{X}-100$ and incubated $\left(24\right.$ hours, $\left.37^{\circ} \mathrm{C}\right)$ in zymography buffer $\left(50 \mathrm{mM}\right.$ Tris $\mathrm{HCl}, 10 \mathrm{mM} \mathrm{CaCl}_{2}$, $50 \mathrm{mM} \mathrm{NaCl}, 0.05 \%$ BRIJ 35). Gels were stained using Coomassie Brilliant Blue R-250 (Sigma Chemical Co.). Clear areas on the Coomassie-stained gel indicated sites of gelatinolysis.

Immunohistochemistry. Atherosclerotic plaques from human coronary and carotid arteries $(n=12)$ and nonatherosclerotic arteries $(n=5)$ were obtained from transplantation donors, at endarterectomy and at autopsy. Air-dried serial cryostat sections $(6 \mu \mathrm{m})$ were fixed in acetone $\left(5\right.$ minutes, $\left.-20^{\circ} \mathrm{C}\right)$, preincubated with $\mathrm{PBS} / 0.3 \% \mathrm{H}_{2} \mathrm{O}_{2}$, and incubated (30 minutes) with primary or control $\mathrm{Ab}$ (mouse myeloma protein MOPC-21; Sigma Chemical Co.; or rabbit IgG). Subsequent processing was performed according to the manufacturer's recommendations (Universal DAKO LSAB peroxidase kit; DAKO Corp., Carpinteria, California, USA). Ab binding was visualized with 3 -amino9-ethyl carbazole (AEC; Sigma Chemical Co.). For colocalization of TFPI-2 with the respective cell types or MMPs, double-immunofluorescence staining was performed. Frozen sections were incubated with rabbit anti-human TFPI-2 Ab (90 minutes), followed by biotinylated secondary goat anti-rabbit $\mathrm{Ab}$ (45 minutes; Vector Laboratories, Burlingame, California, USA) and streptavidin conjugated with Texas red (30 minutes; Amersham Pharmacia Biotech Inc.). Subsequently, specimens were treated with an avidinbiotin-blocking kit (Vector Laboratories), washed, and stained with monoclonal cell type- or MMP-specific Abs $\left(24\right.$ hours, $\left.4^{\circ} \mathrm{C}\right)$, biotinylated secondary horse anti-mouse $\mathrm{Ab}$, and finally FITC-conjugated streptavidin (30 minutes) (Amersham Pharmacia Biotech Inc.). Nuclei were stained blue with bisbenzimide (Calbiochem-Novabiochem Corp.). 


\section{Results}

TFPI-2 inhibits MMP activity. To determine whether TFPI-2 inhibits MMP activity directly, varying concentrations of human recombinant TFPI-2 were preincubated with activated recombinant MMP-1 (1 hour, $37^{\circ} \mathrm{C}$ ) before addition of fluorophore-conjugated native type I collagen substrate. TFPI- 2 concentration dependently inhibited MMP-1-mediated $(10 \mu \mathrm{g} / \mathrm{ml}$, corresponding to $200 \mathrm{nM}$ MMP-1) collagenolysis (Figure $1 \mathrm{a})$ with a median effective dose $\left(\mathrm{EC}_{50}\right)$ of $1.6 \pm 0.53$ $\mu \mathrm{g} / \mathrm{ml}$, corresponding to approximately $57.6 \pm 19.1 \mathrm{nM}$ TFPI-2 $(n=10)$. Maximal inhibition $(85.9 \% \pm 0.23 \%$; $n=10)$ was observed with $9.6 \pm 5.2 \mu \mathrm{g} / \mathrm{ml}$ TFPI-2 $(345 \pm 187 \mathrm{nM})$. Since we recently provided evidence for interstitial collagenolysis by MMP-13 within human atheroma (9), we analyzed TFPI-2's effect on this MMP as well. TFPI- 2 diminished MMP-13 activity $(10 \mu \mathrm{g} / \mathrm{ml})$ in a stoichiometric fashion similar to that observed for MMP-1, achieving similar maximal inhibition (Figure $1 \mathrm{~b}$ ) with an $\mathrm{EC}_{50}$ of $0.876 \pm 261$ $\mu \mathrm{g} / \mathrm{ml}$, corresponding to approximately $31.5 \pm 9.4 \mathrm{nM}$ TFPI-2 $(n=4)$. TFPI-2 and TIMP-1 had similar inhibitory stoichiometry (Figure 1, e and f). It remains to be determined whether the incomplete inhibition of MMP activity by TFPI-2 is due to insufficient inhibitory capability or to assay background, although the finding that TIMP-1 in the same assays yielded similar maximal inhibition supports the latter explanation. We investigated further whether TFPI-2 inhibited downstream mediators of collagenolysis. This serpin inhibited the gelatinases MMP-2 and MMP-9 less effectively and potently than it did interstitial collagenases (Figure 1, c and d). Maximal inhibition of the gelatinases $(10 \mu \mathrm{g} / \mathrm{ml})$ required $58.9 \pm 17.1 \mu \mathrm{g} / \mathrm{ml}(2.1 \pm 0.6$ $\mu \mathrm{M})$ and $63.4 \pm 20.0 \mu \mathrm{g} / \mathrm{ml}(2.3 \pm 0.7 \mu \mathrm{M})$, lowering activity of MMP- 2 to $40 \% \pm 14.6 \%(n=6)$ and of MMP-9 to $37 \% \pm 14.4 \%(n=6)$, respectively. The median effective concentration corresponded to $15.3 \pm 3.4 \mu \mathrm{g} / \mathrm{ml}$ $(551 \pm 122 \mathrm{nM})$ and $14.1 \pm 4.8 \mu \mathrm{g} / \mathrm{ml}(508 \pm 173 \mathrm{nM})$ TFPI-2 for MMP-2 and MMP-9, respectively. A similarly effective inhibition of gelatinase required a molar ratio of 3:1 of TIMP-1/MMP (data not shown).

Control experiments demonstrated inactivity of MMP-2 and MMP-9 in the collagenase assay (data not shown). Moreover, nonactivated MMP-1 or MMP-13 did not exhibit gelatinolytic activity, eliminating the possibility that other enzymes, e.g., serine proteinases, could account for the ECM-degrading activity provided by the MMP-preparations employed. APMA-activated MMP-1 and MMP-13 did have some gelatinolytic activity, though considerably less than MMP-2 or MMP-9 (data not shown). Moreover, TFPI-1 did not affect MMP activity, and TFPI-2 alone did not affect fluorescence intensity or optical density in any of the assays used (data not shown). To determine the specificity of TFPI-2-mediated inhibition, cathepsins $S$ and $\mathrm{K}$ were applied to processing assays identical to those employed for MMPs, but using elastin as the native substrate. TFPI-2 did not affect the elastolytic activity of either cathepsin, even at concentrations as high as $50,000 \mathrm{ng} / \mathrm{ml}$ (Figure 1g).

To explore further the capacity of TFPI-2 to diminish collagenolysis, the degradation of triple-helical type I collagen by either MMP-1 or MMP-13 preincubated with or without the serpin was analyzed by Western blotting. Treatment of interstitial collagen with these MMPs yielded the expected three-quarter-length cleavage product and loss of the immunoreactive full-length type I collagen. Similar to the fluorogenic assays, TFPI-2 concentration dependently inhibited the cleavage of type I

\section{Figure 3}

Differential expression of TFPI-2 in cultured SMCs and MØs. (a) Human vascular SMCs were cultured in IT medium (24 hours) and either stimulated 24 hours with the respective concentrations of IL-1 $\beta /$ TNF- $\alpha$ (left), or stimulated for the indicated times with $10 / 50$ $\mathrm{ng} / \mathrm{ml} \mathrm{IL-1 \beta /TNF- \alpha} \mathrm{(middle)} \mathrm{or} 10 \mu \mathrm{g} / \mathrm{ml}$ CD40L (right). (b) Mononuclear phagocytes $(M \varnothing)$ were cultured for either $0,1,3$, or 11 days in RPMI-1640 medium supplemented with $5 \%$ human serum. Before (24 hours) stimulation with LPS $(100 \mathrm{ng} / \mathrm{ml})$, cells were cultured in serum-free medium. SMC and M $\varnothing$ culture lysates $(30 \mu \mathrm{g} /$ lane $)$ and SMC supernatants (SN) were applied to Western blot analysis employing $\alpha$-TFPI-2. The positions of the molecular-weight markers are indicated on the left. Comparable data were obtained employing cells from four different donors.

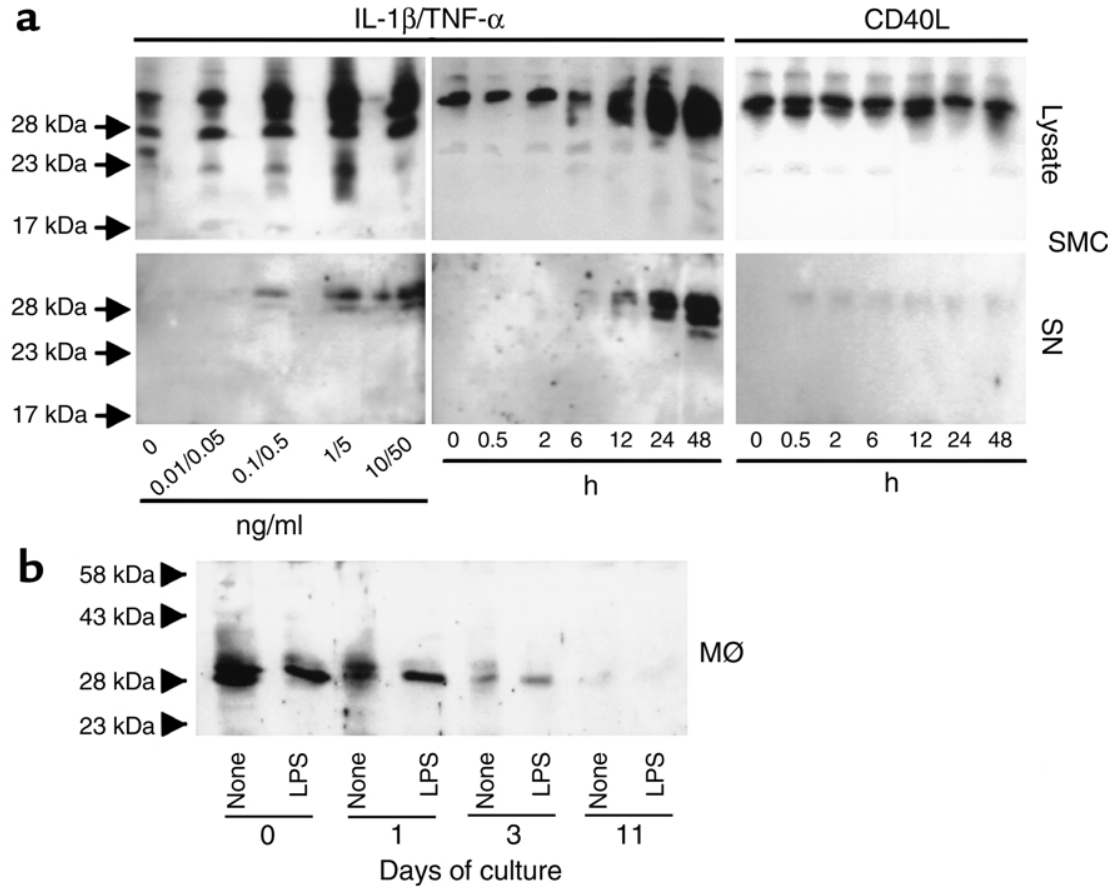



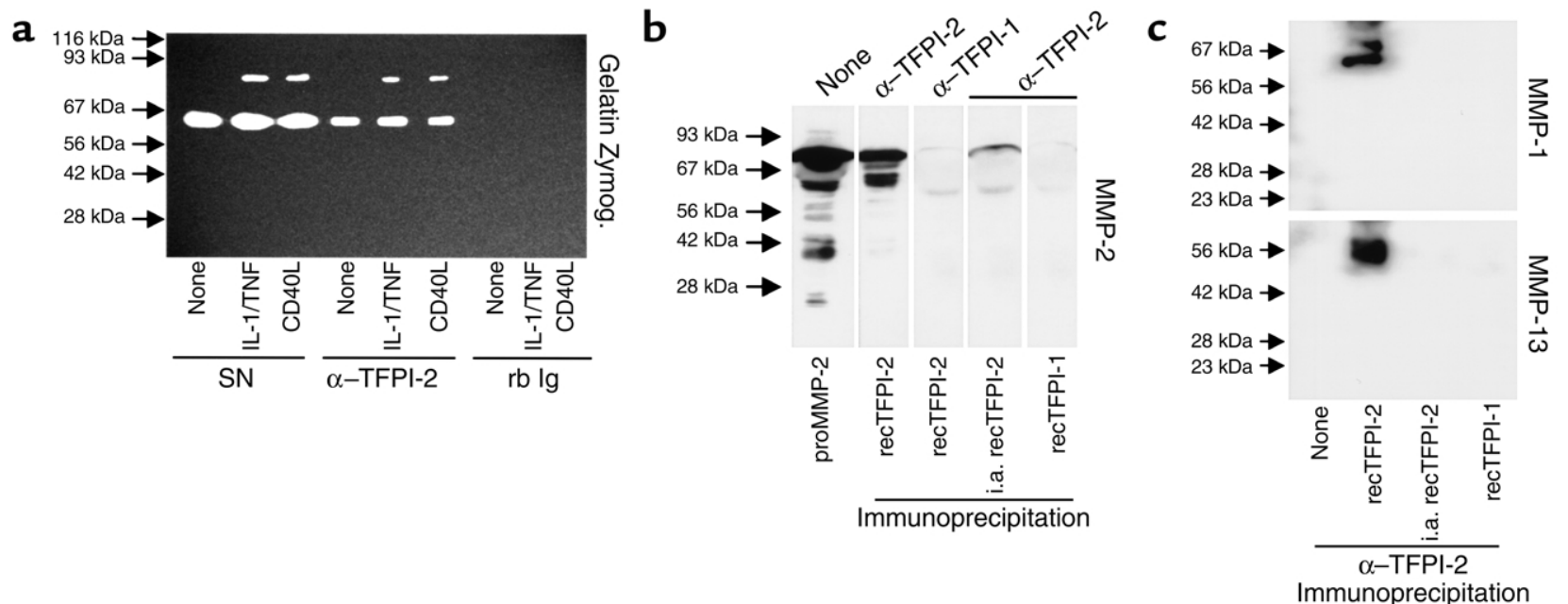

\begin{abstract}
Figure 4
Anti-TFPI-2 coprecipitates MMP-1, MMP-2, MMP-9, and MMP-13. (a) Supernatants of unstimulated (none), IL-1 $\beta /$ TNF- $\alpha$-stimulated $(10 / 50 \mathrm{ng} / \mathrm{ml})$, or CD40L-stimulated $(10 \mu \mathrm{g} / \mathrm{ml})$ human vascular SMCs were immunoprecipitated with anti-TFPI-2 ( $\alpha$-TFPI-2) or rabbit $\lg (\mathrm{rb}$ lg), and the precipitates as well as the original culture supernatants (SN) were analyzed by SDS-PAGE zymography. Clear areas indicate gelatinolysis. Comparable data were obtained employing SMCs from three different donors. (b) Recombinant MMP-2 precursor as well as (c) active MMP-1 (top) and MMP-13 (bottom) (all $10 \mu \mathrm{g} / \mathrm{ml}$ ) were incubated ( 1 hour, $37^{\circ} \mathrm{C}$ ) with recombinant TFPI-2 (50 $\mu \mathrm{g} / \mathrm{ml}$ ). Reactions were immunoprecipitated with anti-TFPI-2 and analyzed by Western blotting for the respective MMP. For control purposes heatinactivated recombinant TFPI-2 (i.a. recTFPI-2), recombinant TFPI-1 $(50 \mu \mathrm{g} / \mathrm{ml})$, or $\alpha$-TFPI-1 was applied. The positions of the molecular-weight markers are indicated on the left.
\end{abstract}

collagen by both interstitial collagenases (Figure 2). As already demonstrated in the fluorogenic assays, TFPI-2 inhibited both collagenases with stoichiometry similar to TIMP-1 (Figure 2, a and b). Interestingly, preincubating TFPI-2 with VIIa/TF (Figure 2) or with trypsin (data not shown), did not diminish the inhibitory capacity of the serpin against MMP-1 or MMP-13 in either the purified (Figure 2c) or fluorogenic (Figure 2d) type I collagen assay, indicating that inhibition of MMPs and serine proteinases employs different domains of TFPI-2.

Human vascular SMCs and MØs differentially express TFPI- 2 in vitro. Further experiments analyzed the expression of TFPI- 2 by SMCs and MØs, established sources of MMPs in human atheroma. Cultured human vascular SMCs expressed cell-associated TFPI-2 constitutively (Figure 3, top), with the majority of the inhibitor localizing in the ECM (data not shown), as previously reported for EC (4). Stimulation with the inflammatory cytokines IL- $1 \beta$ and TNF- $\alpha$ concentration and time dependently enhanced the constitutive cell-associated expression of TFPI- 2 and furthermore induced the release of the inhibitor. In contrast, another potent agonist for MMP expression in SMCs, CD40L, did not appreciably induce either cell-associated TFPI-2 or release of the inhibitor, even in concentrations up to 10 $\mu \mathrm{g} / \mathrm{ml}$ (Figure 3, top). None of these stimuli affected cell number or viability (data not shown).

Since MØs probably comprise the major source of MMPs in human atheroma, we examined TFPI-2 expression by this cell type as well. Stimulation with IL-1 $\beta$, TNF- $\alpha$, CD40L (data not shown), or LPS (Figure 3b) did not sub- stantially affect the constitutive expression of TFPI- 2 in freshly isolated peripheral blood mononuclear phagocytes. Culture of these cells for 3 days diminished expression of immunoreactive TFPI-2, which became undetectable after prolonged culture. Thus, regulation of this endogenous inhibitor inversely correlates to the expression of the interstitial collagenases MMP- 1 and MMP-13 during M $\varnothing$ differentiation, as published previously (9).

To investigate potential mechanisms of TFPI-2-mediated inhibition of MMP activity, SMC culture supernatants were immunoprecipitated with anti-TFPI- $2 \mathrm{Ab}$. Indeed, anti-TFPI-2 coprecipitated gelatinolytic activity with an apparent molecular weight of $72 \mathrm{kDa}$ and 92 $\mathrm{kDa}$, corresponding to the expected molecular weight of MMP-2 and MMP-9, demonstrating protein/protein interactions between TFPI-2 and MMPs (Figure 4a). Control nonimmune rabbit IgG did not coprecipitate gelatinolytic activity, demonstrating specificity of the Ab employed (Figure 4a). Moreover, anti-TFPI-2 coprecipitated the MMP-2 zymogen (Figure 4b), as well as mature forms of the interstitial collagenases MMP-1 and MMP-13 (Figure 4c) after cell-free coincubation in vitro of recombinant TFPI- 2 with the respective recombinant MMP, demonstrating direct interaction between enzyme and inhibitor. Control experiments showed that heat-inactivated $\left(10\right.$ minutes, $\left.95^{\circ} \mathrm{C}\right) \mathrm{TFPI}-2$ lost the ability to interact with the MMPs tested. Furthermore, anti-TFPI-2 did not precipitate either MMP directly or via TFPI-1 (Figure 4, $b$ and $c$ ).

TFPI-2 colocalizes with SMCs, but not MØs, in atheromatous lesions in situ. In accordance with the differential in vitro 
regulation of TFPI-2 expression in SMCs and MØs, cells considered major producers of MMPs within human atheroma, we also observed differential expression of this endogenous inhibitor in these cell types in atherosclerotic lesions in situ. Immunohistochemical analysis demonstrated that TFPI- 2 colocalizes with SMCs in both the tunica media of normal arteries and the media beneath the fibrous cap overlying atheromatous lesions (Figure 5, a and b). Staining for the inhibitor in SMCs within diseased tissue appeared more intense than staining in the nonatherosclerotic media (data not shown). In contrast, MØ-enriched areas, such as the shoulder region of the plaque, stained weakly, if at all, for TFPI-2 (Figure 5c). TFPI-2 further colocalized with ECs, an established source for this Kunitz-type inhibitor (4), with microvascular EC exhibiting particularly intense staining (data not shown).

Because TFPI-2 coprecipitated with MMPs in vitro (Figure 4), we further investigated whether MMPs and TFPI-2 colocalize in situ. Although MMP-2 (Figure 6a) and MMP-9 (Figure 6b) colocalized with TFPI-2, the proteinases localized predominantly within the MØenriched shoulder region, whereas TFPI-2 mostly localized in the SMC-enriched fibrous cap of the lesion, as determined by immunofluorescence doublestaining (Figure 6c). As with the gelatinases, immunoreactive MMP-1 (red, Figure $6 \mathrm{~d}$ ) and MMP-13 (red, Figure 6e) exceeded levels of TFPI-2 (green) in the MØ-enriched area of the lesion (right panels), in contrast to the prominent presence of the inhibitor in the SMC-enriched fibrous cap (left panels). These findings demonstrate differential expression of TFPI- 2 and MMPs within human atherosclerotic lesions, with the inhibitor predominant in the fibrous cap and sparse in areas associated with plaque instability and rupture, although immunohistochemical analysis does not permit strict quantitation. This finding has particularly important implications with respect to the activity of MMP-1 and MMP-13, enzymes we recently implicated in collagenolysis within human atheroma (9).

In support of these in situ observations, extracts from normal arteries contained more TFPI- 2 than did fibrous plaques, while extracts of atheromatous lesions with morphologic features of vulnerability expressed even less TFPI-2. In contrast, atherosclerotic plaques exhibited more immunoreactive TIMP-1 and TIMP-2 than nondiseased arteries (Figure 6f). Thus, expression of TFPI-2, but not of TIMP-1 or TIMP-2, correlates inversely with the expression of the interstitial collagenases MMP-1 and MMP-13 during plaque progression (9).

\section{Discussion}

Matrix turnover critically determines the progress of various diseases, such as cancer, arthritis, and atherosclerosis $(6,11,40,41)$. Matrix-degrading enzymes, such as MMPs, can control ECM content during tumor metastasis, cartilage deterioration, and arterial lesion development and complications. Though mechanisms regulating the enzymatic activity of these proteinases have major medical ramifications, the processes determining MMP activity remain incompletely understood. Previous work by us and others demonstrated that proinflammatory cytokines, such as TNF- $\alpha$ and IL-1 $\beta$, as well as CD40L, induce the expression of MMPs from atheroma-associated cells $(10,12-16,42)$. Enzymatic function requires activation of MMP zymogens, probably stimulated by autocatalysis, small molecules (e.g., superoxide, peroxynitrite, or hydrogen peroxide), or proteases (e.g., thrombin, plasmin, or MT-MMPs) (43-46). However, the expression and release of active MMP molecules does not establish matrix-degrading activity, since interactions with ubiquitous endogenous inhibitors determine enzymatic function in tissues. In this regard, most attention has focused on the TIMP family (19-25). However, previous work from several groups also implicated members of the serpin superfamily, i.e., $\alpha_{2}$-macroglobulin and RECK, in the regula-

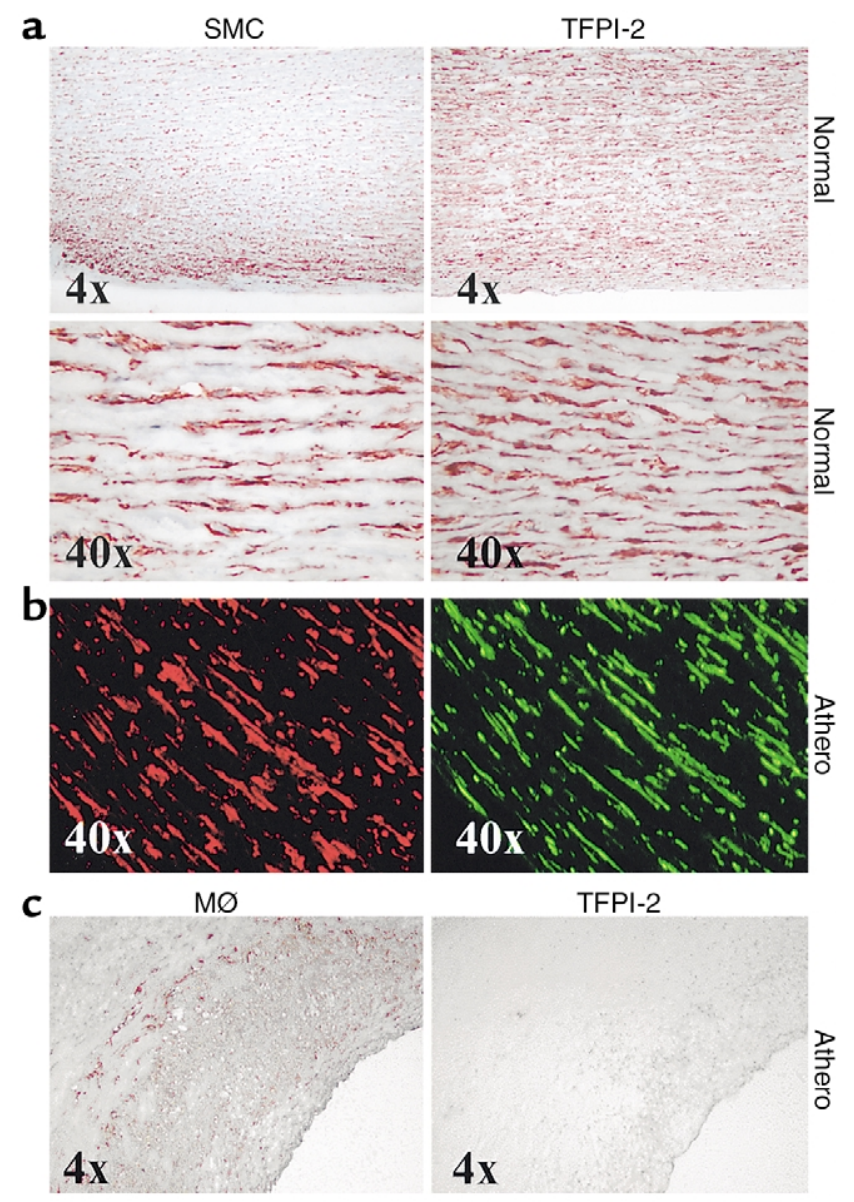

Figure 5

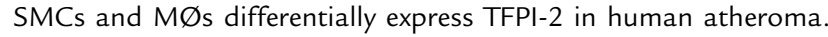
Cryostat sections from nondiseased aortae (Normal) and atherosclerotic carotid atheroma (Athero) were stained for SMCs and MØs employing (a, b) anti- $\alpha$-actin and (c) $\alpha$-CD68, respectively (left panels). Localization of TFPI-2 (right panels) was shown by $(\mathbf{a}, \mathbf{c})$ light microscopy in adjacent sections, or (b) immunofluorescence double labeling for direct colocalization within the same section ( $\alpha$-actin staining for SMCs in red; TFPI-2 staining in green). Analysis of surgical specimens obtained from four different donors showed similar results. 


\section{Figure 6}

TFPI-2 expression is diminished in the shoulder region of human atherosclerotic plaques. $(\mathbf{a}, \mathbf{b})$ Shown are single-wavelength filter photomicrographs $(\times 10)$ of cryostat sections from atherosclerotic carotid atheroma applied to double-immunofluorescence labeling to colocalize (a) MMP-2 or (b) MMP-9 (both red) with TFPI-2 (green) within the SMC-enriched fibrous cap. (c) Triple-immunofluorescence labeling colocalized MMP-2 (red) with TFPI-2 (green) within the plaque's shoulder region (left: $\times 4$, right: $\times 10$ ). Nuclei are stained in blue. High-magnification photomicrographs $(\times 40)$ demonstrated differential expression of (d) MMP-1 or (e) MMP-13 (both red) with TFPI-2 (green) in SMC-enriched (left) or M $\varnothing$-enriched (right) areas of human atheroma; nuclei are stained in blue. (f) Protein extracts from frozen tissue of nonatherosclerotic carotid arteries (Normal), as well as carotid plaques dichotomized into lesions displaying features associated with either stable (Fibrous) or vulnerable (Athero) plaques, were analyzed by Western blotting employing $\alpha$-TIMP-1 (top), -TIMP-2 (middle), or -TFPI-2 (bottom). Analysis of five normal arteries, five fibrous and seven atheromatous surgical specimens of atheroma from different donors showed similar results.

tion of MMP activity (33-35). The present report demonstrates inhibition of active MMPs by the serpin TFPI-2, probably by direct protein/protein interactions, employing domains distinct from those responsible for serine-proteinase inhibition. Moreover, the findings presented here support the hypothesis that matrix-bound TFPI-2 in nondiseased tissue favors ECM stability by inactivating MMPs, and vice versa, diminished TFPI-2 might allow increased matrix degradation in plaques, enhancing their susceptibility to rupture. Accordingly, nonatherosclerotic arterial tissue and its predominant cell type, vascular SMCs, constitutively expressed TFPI- 2 in situ and in vitro. Thus, SMC might contribute to the integrity of the vascular wall in a dual fashion by functioning as the major source of $\operatorname{ECM}(47,48)$ and expressing the endogenous MMP inhibitor TFPI-2. The prominent binding of the serpin to the ECM probably raises its local effective concentration. The enhanced TFPI- 2 staining of individual SMCs within the fibrous cap compared with those in the media of nondiseased arteries agrees with the in vitro inducibility of the inhibitor by atheromaassociated cytokines such as IL- $1 \beta$ and TNF- $\alpha$. Enhanced expression as well as inducible release of TFPI-2 in response to inflammatory cytokines might function as additional protection to the SMC-generated ECM at sites of inflammation.

Furthermore, in accordance with our hypothesis that lack of TFPI- 2 contributes to enhanced matrix degradation in human atheroma, this report demonstrates diminished TFPI-2, but not TIMP, levels in atheromatous plaques, lesions that contain abundant MMPs (9). Whether this indeed indicates a more important role for the serpin than TIMPs in the regulation of MMP activity remains to be determined, since compartmentalization might result in distinct microenvironments with corresponding variations in $\mathrm{MMP} /$ inhibitor ratios. The relative paucity of TFPI- 2 in situ in the shoulder of the a
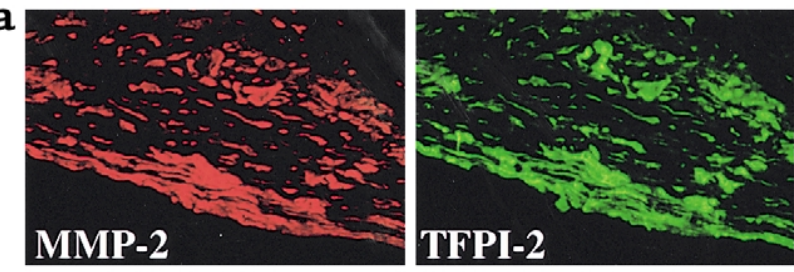

b
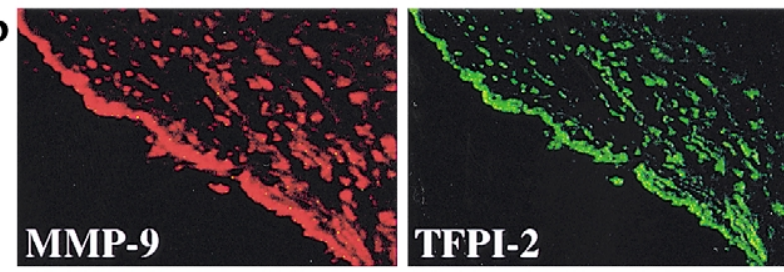

c
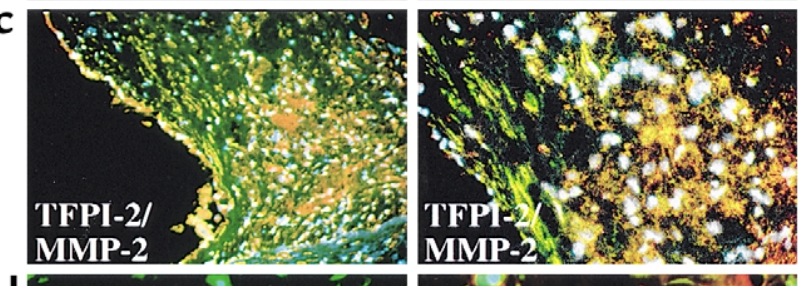

d 5

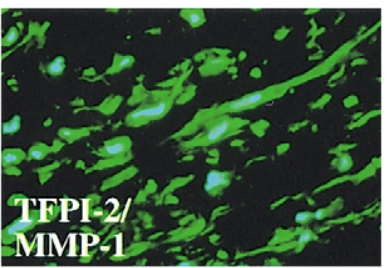

e 2
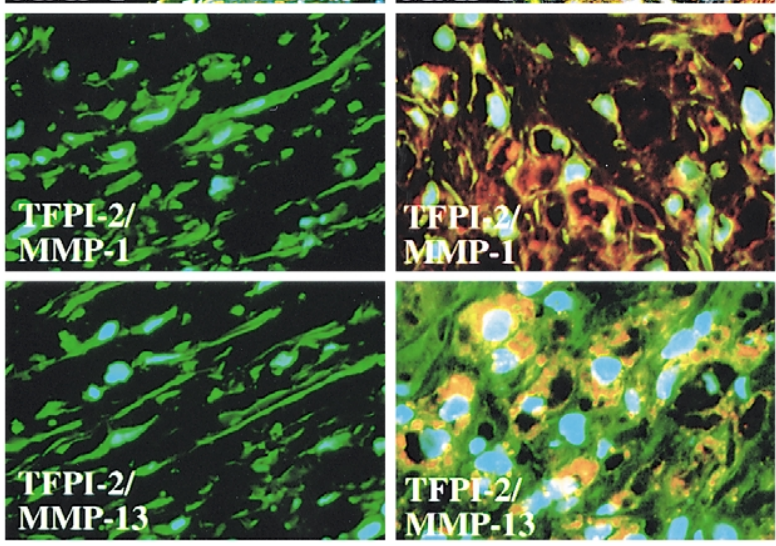

f

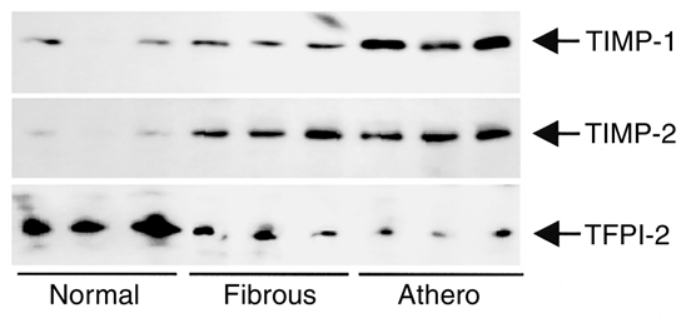

plaque, a region characterized by enhanced collagenolysis as well as enhanced expression of MMP-1 and MMP-13 (9), may be explained by the diminished expression of TFPI- 2 during differentiation of MØ. This part of the plaque experiences the highest circumferential stress and is particularly prone to rupture (49). Inhibition of interstitial collagenases by TFPI-2 has particular bearing on plaque stability, as these enzymes are required for the initial step of degradation. In this regard, the role of MMP-2 as a collagenase (50), a finding disputed by other groups (51), remains uncertain, since we did not observe collagenolytic activity for either gelatinase in our collagenolytic assays. Further supporting our hypothesis that TFPI-2/MMP imbalance crucially determines plaque stability, MØs rather than 
SMCs appear to elaborate the bulk of the matrixdegrading enzymes, particularly interstitial collagenases, in human atheroma $(9,10,52-54)$. Aside from the diminished expression in differentiated macrophages, decreased levels of total immunoreactive TFPI- 2 in plaques probably result from fewer numbers of SMCs rather than diminished expression of TFPI-2 per SMC because (a) cytokines present in atheroma increase TFPI- 2 expression by SMC in vitro, and (b) accordingly, TFPI-2 expression in situ is actually increased in SMCs within the fibrous cap of human atheroma compared with that in nondiseased arterial tissue.

Demonstrating that TFPI-2 limits the enzymatic activity of MMPs also suggests a mechanism by which TFPI-2 reduced matrix degradation by highly invasive fibrosarcoma cells, which could not penetrate Matrigel in the presence of this serpin (36). Thus, these findings indicate a new biological role for TFPI- 2 as an inhibitor of matrix degradation in atherosclerosis as well as other chronic inflammatory diseases, such as cancer.

A previous study indicating that TFPI- 2 might participate in matrix-protective processes described inhibition of plasmin-dependent activation of MMP zymogens by TFPI-2 (5). However, the mechanism remained unclear. The present data established that TFPI-2 and MMPs can interact by direct protein/protein interactions, probably involving sites of interaction distinct from those responsible for the inhibition of serine proteinases. In addition to inhibiting plasmin directly, the serpin TFPI-2 might therefore prevent plasmin-dependent activation by binding to proMMPs, either sterically hindering plasmin or altering the MMP zymogen allosterically.

Furthermore, the surprising finding that the serpin TFPI-2 inhibits nonserine proteinases might be explained by potential molecular mechanism of inhibition resembling those acting in TIMPs. TIMPs are comprised of two domains, each containing three disulfide bonds thought to coordinate the requisite $\mathrm{Zn}^{+}$ion in the MMP active site (23). Similarly, TFPI-2 contains three domains that also contain three disulfide bonds each, possibly providing a similar inhibitory mechanism.

In summary, the observed expression of TFPI- 2 in SMCs of normal and diseased tissue and diminished expression of the inhibitor in lesional $\mathrm{M} \varnothing$, in accordance with the in vitro data, supports the hypothesis that this serpin limits ECM degradation in the human vascular wall and that the loss of TFPI-2 might promote plaque formation and/or complication. Whether TFPI-2 acts in concert with other endogenous MMP inhibitors, such as TIMPs, and whether TFPI- 2 indeed crucially regulates MMP activity in vivo will require further study.

\section{Acknowledgments}

This work was supported in part by grants of the National Heart, Lung, and Blood Institute (HL-56985 to P. Libby) and performed during the tenure of $U$. Schönbeck as the Paul Dudley White Fellow of the
American Heart Association. We thank Maria Muszynski, Eugenia Shvartz, Anna Papautsky, and Elissa Simon-Morrissey (Brigham and Women's Hospital) for skillful technical assistance, Guo Ping-Shi and Carlos Lopez-Otin for kindly providing reagents, and Karen Williams for editorial assistance.

1. Sprecher, C.A., Kisiel, W., Mathewes, S., and Foster, D.C. 1994. Molecular cloning, expression, and partial characterization of a second human tissue-factor-pathway inhibitor. Proc. Natl. Acad. Sci. USA. 91:3353-3357.

2. Petersen, L.C., et al. 1996. Inhibitory properties of a novel human Kunitz-type protease inhibitor homologous to tissue factor pathway inhibitor. Biochemistry. 35:266-272.

3. Butzow, R., Huhtala, M.L., Bohn, H., Virtanen, I., and Seppala, M. 1988. Purification and characterization of placental protein 5. Biochem. Biophys. Res. Commun. 150:483-490.

4. Iino, M., Foster, D.C., and Kisiel, W. 1998. Quantification and characterization of human endothelial cell-derived tissue factor pathway inhibitor-2. Arterioscler. Thromb. Vasc. Biol. 18:40-46.

5. Rao, C.N., Mohanam, S., Puppala, A., and Rao, J.S. 1999. Regulation of ProMMP-1 and ProMMP-3 activation by tissue factor pathway inhibitor$2 /$ matrix-associated serine protease inhibitor. Biochem. Biophys. Res. Commun. 255:94-98.

6. Newby, A.C., Southgate, K.M., and Davies, M. 1994. Extracellular matrix degrading metalloproteinases in the pathogenesis of arteriosclerosis. Basic Res. Cardiol. 89(Suppl.):59-70.

7. Morton, L.F., and Barnes, M.J. 1982. Collagen polymorphism in the normal and diseased blood vessel wall. Investigation of collagens types I, III and V. Atherosclerosis. 42:41-51.

8. Stary, H.C. 1990. The sequence of cell and matrix changes in atherosclerotic lesions of coronary arteries in the first forty years of life. Eur. Heart J. 11(Suppl.):3-19.

9. Sukhova, G.K., et al. 1999. Evidence for increased collagenolysis by interstitial collagenases- 1 and -3 in vulnerable human atheromatous plaques. Circulation. 99:2503-2509.

10. Shah, P.K., et al. 1995. Human monocyte-derived macrophages induce collagen breakdown in fibrous caps of atherosclerotic plaques. Potential role of matrix-degrading metalloproteinases and implications for plaque rupture. Circulation. 92:1565-1569.

11. Nagase, H., and Woessner, J.F. 1999. Matrix metalloproteinases. J. Biol. Chem. 274:21491-21494.

12. Schönbeck, U., et al. 1997. Regulation of matrix metalloproteinase expression in human vascular smooth muscle cells by $\mathrm{T}$ lymphocytes: a role for CD40 signaling in plaque rupture? Circ. Res. 81:448-454.

13. Schönbeck, U., et al. 1999. Expression of stromelysin-3 in atherosclerotic lesions: regulation via CD40-CD40 ligand signaling in vitro and in vivo. J. Exp. Med. 189:843-853.

14. Malik, N., Greenfield, B.W., Wahl, A.F., and Kiener, P.A. 1996. Activation of human monocytes through CD40 induces matrix metalloproteinases. J. Immunol. 156:3952-3960.

15. Saren, P., Welgus, H.G., and Kovanen, P.T. 1996. TNF-alpha and IL-1beta selectively induce expression of $92-\mathrm{kDa}$ gelatinase by human macrophages. J. Immunol. 157:4159-4165.

16. Galis, Z.S., et al. 1994. Cytokine-stimulated human vascular smooth muscle cells synthesize a complement of enzymes required for extracellular matrix digestion. Circ. Res. 75:181-189.

17. Nagase, H. 1997. Activation mechanisms of matrix metalloproteinases. Biol. Chem. 378:151-160.

18. Murphy, G., et al. 1999. Mechanisms for pro matrix metalloproteinase activation. APMIS. 107:38-44.

19. Docherty, A.J., et al. 1985. Sequence of human tissue inhibitor of metalloproteinases and its identity to erythroid-potentiating activity. Nature. 318:66-69.

20. Boone, T.C., Johnson, M.J., De Clerck, Y.A., and Langley, K.E. 1990. cDNA cloning and expression of a metalloproteinase inhibitor related to tissue inhibitor of metalloproteinases. Proc. Natl. Acad. Sci. USA. 87:2800-2804

21. Silbiger, S.M., Jacobsen, V.L., Cupples, R.L., and Koski, R.A. 1994. Cloning of cDNAs encoding human TIMP-3, a novel member of the tissue inhibitor of metalloproteinase family. Gene. 141:293-297.

22. Olson, T.M., et al. 1998. Cloning of the human tissue inhibitor of metalloproteinase-4 gene (TIMP4) and localization of the TIMP4 and TIMP4 genes to human chromosome 3 p 25 and mouse chromosome 6 , respectively. Genomics. 51:148-151.

23. Gomis-Ruth, F.X., et al. 1997. Mechanism of inhibition of the human matrix metalloproteinase stromelysin-1 by TIMP-1. Nature. 389:77-81.

24. Brew, K., Dinakarpandian, D., and Nagase, H. 2000. Tissue inhibitors of metalloproteinases: evolution, structure and function. Biochim. Biophys. Acta. 1477:267-283. 
25. Docherty, A.J., and Murphy, G. 1990. The tissue metalloproteinase family and the inhibitor TIMP: a study using cDNAs and recombinant proteins. Ann. Rheum. Dis. 49:469-479.

26. Zaltsman, A.B., George, S.J., and Newby, A.C. 1999. Increased secretion of tissue inhibitors of metalloproteinases 1 and 2 from the aortas of cholesterol fed rabbits partially counterbalances increased metalloproteinase activity. Arterioscler. Thromb. Vasc. Biol. 19:1700-1707.

27. Fabunmi, R.P., Sukhova, G.K., Sugiyama, S., and Libby, P. 1998. Expression of tissue inhibitor of metalloproteinases- 3 in human atheroma and regulation in lesion-associated cells: a potential protective mechanism in plaque stability. Circ. Res. 83:270-278.

28. Strongin, A.Y., et al. 1995. Mechanism of cell surface activation of 72 $\mathrm{kDa}$ type IV collagenase. Isolation of the activated form of the membrane metalloprotease. J. Biol. Chem. 270:5331-5338.

29. Clark, I.M., Powell, L.K., and Cawston, T.E. 1994. Tissue inhibitor of metalloproteinases (TIMP-1) stimulates the secretion of collagenase from human skin fibroblasts. Biochem. Biophys. Res. Commun. 203:874-880.

30. Dean, D.D., Martel-Pelletier, J., Pelletier, J.P., Howell, D.S., and Woessner, J.F., Jr. 1989. Evidence for metalloproteinase and metalloproteinase inhibitor imbalance in human osteoarthritic cartilage. J. Clin. Invest. 84:678-685.

31. Owen, C.A., and Campbell, E.J. 1999. The cell biology of leukocyte-mediated proteolysis. J. Leukoc. Biol. 65:137-150.

32. Rice, W.G., and Weiss, S.J. 1990. Regulation of proteolysis at the neutrophil-substrate interface by secretory leukoprotease inhibitor. Science. 249:178-181.

33. Werb, Z., Burleigh, M.C., Barrett, A.J., and Starkey, P.M. 1974. The interaction of alpha2-macroglobulin with proteinases. Binding and inhibition of mammalian collagenases and other metal proteinases. Biochem. J. 139:359-368.

34. Woessner, J.F., Jr. 1999. Matrix metalloproteinase inhibition. From the Jurassic to the third millennium. Ann. NY Acad. Sci. 878:388-403.

35. Takahashi, C., et al. 1998. Regulation of matrix metalloproteinase-9 and inhibition of tumor invasion by the membrane-anchored glycoprotein RECK. Proc. Natl. Acad. Sci. USA. 95:13221-13226.

36. Rao, C.N., et al. 1998. HT-1080 fibrosarcoma cell matrix degradation and invasion are inhibited by the matrix-associated serine protease inhibitor TFPI-2/33 kDa MSPI. Int. J. Cancer. 76:749-756.

37. Pullen, S.S., et al. 1999. High-affinity interactions of tumor necrosis factor receptor-associated factors (TRAFs) and CD40 require TRAF trimerization and CD40 multimerization. Biochemistry. 38:10168-10177.

38. Shi, G.P., Munger, J.S., Meara, J.P., Rich, D.H., and Chapman, H.A. 1992. Molecular cloning and expression of human alveolar macrophage cathepsin S, an elastinolytic cysteine protease. J. Biol. Chem. 267:7258-7262.

39. Libby, P., and O'Brien, K.V. 1983. Culture of quiescent arterial smooth muscle cells in a defined serum-free medium. J. Cell Physiol. 115:217-223.
40. Curran, S., and Murray, G.I. 1999. Matrix metalloproteinases in tumour invasion and metastasis. J. Pathol. 189:300-308.

41. Libby, P., and Lee, R.T. 2000. Matrix matters. Circulation. 102:1874-1876.

42. Mach, F., et al. 1999. T lymphocytes induce endothelial cell matrix metalloproteinase expression by a CD40L-dependent mechanism: implications for tubule formation. Am. J. Pathol. 154:229-238.

43. Rajagopalan, S., Meng, X.P., Ramasamy, S., Harrison, D.G., and Galis, Z.S. 1996. Reactive oxygen species produced by macrophage-derived foam cells regulate the activity of vascular matrix metalloproteinases in vitro. Implications for atherosclerotic plaque stability. J. Clin. Invest. 98:2572-2579.

44. Galis, Z.S., Kranzhofer, R., Fenton, J.W., II, and Libby, P. 1997. Thrombin promotes activation of matrix metalloproteinase- 2 produced by cultured vascular smooth muscle cells. Arterioscler. Thromb. Vasc. Biol. 17:483-489.

45. Nagase, H., Enghild, J.J., Suzuki, K., and Salvesen, G. 1990. Stepwise activation mechanisms of the precursor of matrix metalloproteinase 3 (stromelysin) by proteinases and (4-aminophenyl)mercuric acetate. Biochemistry. 29:5783-5789.

46. Knauper, V., et al. 1996. Cellular mechanisms for human procollagenase3 (MMP-13) activation. Evidence that MT1-MMP (MMP-14) and gelatinase a (MMP-2) are able to generate active enzyme. J. Biol. Chem. 271:17124-17131.

47. Libby, P. 1995. Molecular bases of the acute coronary syndromes. Circu lation. 91:2844-2850.

48. Lafont, A., and Libby, P. 1998. The smooth muscle cell: sinner or saint in restenosis and the acute coronary syndromes? J. Am. Coll. Cardiol. 32:283-285.

49. Lee, R.T., Schoen, F.J., Loree, H.M., Lark, M.W., and Libby, P. 1996. Circumferential stress and matrix metalloproteinase 1 in human coronary atherosclerosis. Implications for plaque rupture. Arterioscler. Thromb. Vasc. Biol. 16:1070-1073.

50. Aimes, R.T., and Quigley, J.P. 1995. Matrix metalloproteinase-2 is an interstitial collagenase. Inhibitor-free enzyme catalyzes the cleavage of collagen fibrils and soluble native type I collagen generating the specific 3/4- and 1/4-length fragments. J. Biol. Chem. 270:5872-5876.

51. Seltzre, J.L., and Eisen, A.Z. 1999. Native type I collagen is not a substrate for MMP2 (gelatinase A). J. Invest. Dermatol. 112:993-994.

52. Galis, Z.S., Sukhova, G.K., Lark, M.W., and Libby, P. 1994. Increased expression of matrix metalloproteinases and matrix degrading activity in vulnerable regions of human atherosclerotic plaques. J. Clin. Invest. 94:2493-2503.

53. Carr, S.C., Farb, A., Pearce, W.H., Virmani, R., and Yao, J.S. 1997. Activated inflammatory cells are associated with plaque rupture in carotid artery stenosis. Surgery. 122:757-763.

54. Moreno, P.R., et al. 1994. Macrophage infiltration in acute coronary syndromes. Implications for plaque rupture. Circulation. 90:775-778. 